\section{ATIP, une nouvelle superfamille de protéines associées aux microtubules}

Angie Molina, Sylvie Rodrigues-Ferreira, Anne Di Tommaso, Clara Nahmias
Inserm, U1016, Institut Cochin, Paris, France;

Cnrs, UMR8104, Paris, France;

Université Paris Descartes, Paris, France.

Département EMC,

22, rue Méchain, 75014 Paris, France.

clara.nahmias@inserm.fr

> Les microtubules, formés de l'assemblage dynamique de dimères d'alpha et de bêta-tubuline, jouent un rôle essentiel dans l'homéostasie cellulaire. Dans les cellules en interphase, le cytosquelette de microtubules intervient dans le maintien de l'architecture cellulaire, la migration et le trafic intracellulaire de protéines et d'organites; en mitose, il se réorganise pour former le fuseau mitotique qui permet une répartition correcte des chromosomes [1]. La dynamique de polymérisation des microtubules, essentielle à leur fonction, est sous le contrôle d'un ensemble de protéines agissant de concert: les MAP (microtubule-associated proteins) dont l'activité est finement régulée dans l'espace et le temps $[1,2]$. Les altérations de la structure ou de la régulation des MAP peuvent avoir des répercussions considérables dans de nombreuses situations physiopathologiques, comme c'est le cas par exemple pour la protéine APC (adenomatous polyposis coli) dans le cancer du

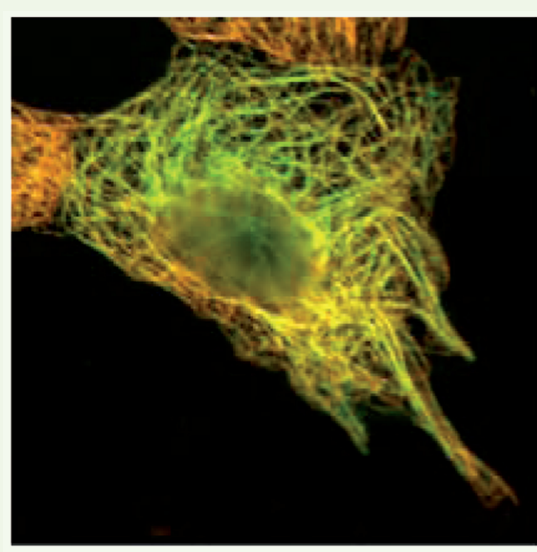

Interphase côlon, ou la protéine Tau dans la maladie d'Alzheimer.

ATIP3, un régulateur de mitose associé aux microtubules

Notre équipe a récemment mis en évidence une nouvelle protéine associée aux microtubules dénommée ATIP3 (AT2-interacting protein 3) [3], codée par le gène candidat suppresseur de tumeurs MTUS1 (microtubule-associated tumor suppressor $)^{1}[4$, 5]. La molécule ATIP3 est localisée au centrosome et le long des microtubules dans les cellules en interphase. Au cours de la division cellulaire, elle s'associe au fuseau mitotique à tous les stades de la mitose et au pont intercellulaire lors de la cytokinèse (Figure 1). En accord avec

1 Le gène MTUSI a initialement été nommé mitochondrial tumor suppressor, sur la base des résultats de Seibold et al. [11]. En 2010, lorsqu'il s'est avéré qu'ATIP3, ICIS et TIP150, produits des gènes MTUS1 et MTUS2, sont associés aux microtubules $[3,7,8]$, le comité de nomenclature de la base de données NCBI (National center for biotechnolog y information) a renommé MTUS1: microtubule-associated tumor suppressor.

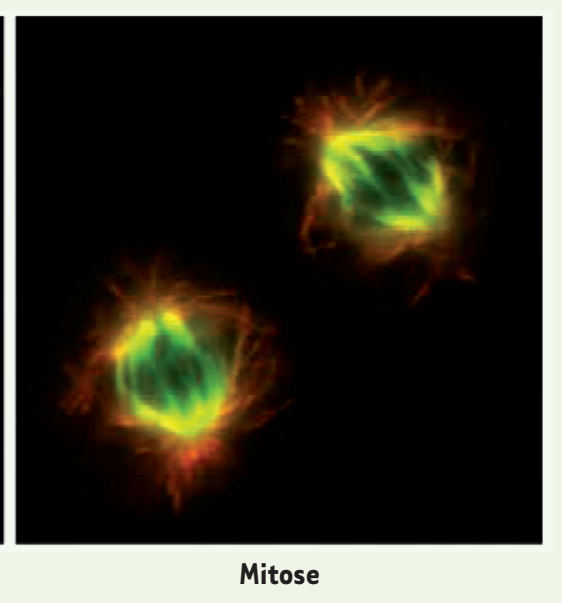

cette localisation particulière, nos travaux ont montré un effet régulateur d'ATIP3 sur la mitose. En effet, ATIP3 freine la prolifération cellulaire et prolonge le temps de division en maintenant les cellules au stade métaphase [3].

L'étude moléculaire de la protéine ATIP3 revêt un intérêt fondamental quand on sait que son expression est diminuée dans le cancer du sein, et ce de façon d'autant plus marquée que la tumeur est plus agressive et de grade histologique élevé, métastatique ou du sous-type triple négatif, pour lequel il n'existe pas à ce jour de thérapie ciblée. Ainsi ATIP3 constitue un nouveau biomarqueur des tumeurs de sein de mauvais pronostic. De plus, ses effets antimitotiques in vitro et antitumoraux in vivo en font une cible privilégiée pour l'élaboration de nouvelles thérapies moléculaires contre le cancer du sein [3].

Bien que les mécanismes d'action d'ATIP3 demeurent inconnus à ce jour, des études récentes réalisées sur deux protéines (ICIS et TIP150) qui lui sont structuralement apparentées, ouvrent des perspectives intéressantes dans le domaine du cancer. La protéine ICIS (codée par le gène orthologue MTUSI de Xénope) a été décrite comme une nouvelle MAP localisée aux

Figure 1. Localisation de la protéine ATIP3 aux microtubules dans les cellules de carcinome pulmonaire SK-MES en interphase (à gauche) et en mitose (à droite). L'image d'immunofluorescence est obtenue après immunomarquage de la protéine ATIP3 endogène (en vert) et de l'alpha-tubuline (en rouge). 


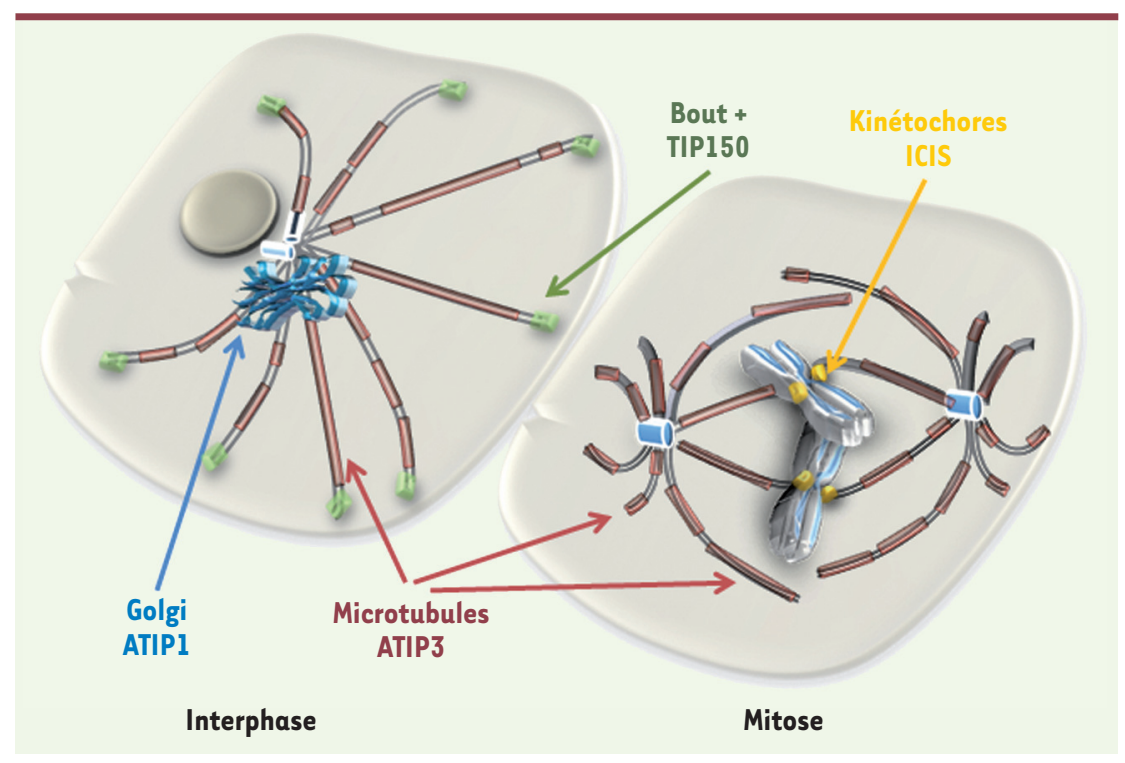

kinétochores [6], structure qui relie les centromères aux microtubules lors de la mitose (Figure 2). ICIS interagit avec la kinésine MCAK (mitotic centromereactivating kinesin) et contribue à la dépolymérisation des microtubules et donc à la dynamique du fuseau mitotique essentiel à la ségrégation des chromosomes [6]. Plus récemment, la mise en évidence d'une interaction entre ICIS et une autre kinésine 13 (Kif2A) conforte le rôle de cette protéine dans la dépolymérisation des microtubules [7]. D'autre part, la protéine TIP150 (produit du gène paralogue MTUS2) a récemment été identifiée comme une MAP interagissant avec $\varepsilon B 1$ (end binding protein 1 ) et MCAK aux bouts « plus » des microtubules (Figure 2) pour favoriser leur dépolymérisation [8]. Ainsi, par analogie avec ses homologues ICIS et TIP150, on peut avancer I'hypothèse d'un rôle d'ATIP3 dans la dynamique des microtubules via l'activation de kinésines de la famille MCAK.

\section{Fonctions}

des autres membres de la famille ATIP Qu'en est-il des autres membres de la famille ATIP ? L'épissage alternatif du gène MTUSI génère en effet deux autres protéines - ATIPI et ATIP4 - identiques à ATIP3 dans leur portion carboxy-terminale $[4,5,9]$ mais dont le lien avec le cytosquelette de microtubules n'a pas encore été évalué. ATIPl, initialement identifié comme partenaire d'interaction du récepteur AT2 de l'angiotensine II - ce qui lui a d'ailleurs valu son nom d'AT2 receptor-interacting protein - est un acteur privilégié des voies de signalisation de ce récepteur, impliqué dans l'inhibition de la prolifération cellulaire, la différenciation neuronale et le remodelage vasculaire [4].

Outre son rôle central dans la signalisation de I'AT2, ATIPl contribue aussi à l'adressage du récepteur à la membrane [10]. De façon intéressante, ATIPI a été décrite comme étant localisée dans les mitochondries [11] ou le Golgi [10], deux compartiments cellulaires étroitement associés aux microtubules. La colocalisation d'ATIPI avec ces deux organites et ses effets sur le trafic intracellulaire du récepteur AT2 suggèrent qu'ATIPl pourrait interagir avec les microtubules et s'associer à des moteurs moléculaires pour permettre le transport intracellulaire d'organites ou de récepteurs. Bien que purement spéculative, cette hypothèse mérite d'être examinée. La protéine ATIP4 n'a pas encore été isolée à ce jour. Au-delà de son domaine d'interaction avec le récepteur AT2, cette protéine présente deux caractéristiques particulières - la présence d'un domaine transmembranaire et un profil d'expression exclusivement restreint au système nerveux central - qui en font un média-

Figure 2. Localisation cellulaire des protéines ATIP et de leurs analogues structuraux ICIS et TIP150. Schéma représentant l'association d'ATIP3, ICIS et TIP150 avec les microtubules dans une cellule en interphase (à gauche) ou en mitose (stade métaphase, à droite). En interphase, ATIP3 (en rose) est localisée tout le long des microtubules alors que TIP150 (en vert) est exclusivement présente aux extrémités des microtubules (bout +). ATIPI (en bleu) est localisée au Golgi. Dans une cellule en mitose, on retrouve ATIP3 (en rose) le long des microtubules du fuseau mitotique et ICIS (en jaune) au niveau des kinétochores.

teur potentiel des effets de l'AT2 dans le cerveau [4]. On peut noter que les 400 acides aminés carboxy-terminaux d'ATIP4 sont identiques à ceux d'ATIPI et ATIP3 et fortement conservés dans les séquences des protéines ICIS et TIP150, ce qui pose la question de l'association potentielle d'ATIP4 avec le cytosquelette de microtubules via son domaine carboxy-terminal intracellulaire.

\section{En conclusion}

Les protéines de la famille ATIP ont récemment été impliquées dans diverses fonctions, allant de la différenciation neuronale au remodelage vasculaire et à la prolifération tumorale. Cette revue pose l'hypothèse selon laquelle ces protéines pourraient, avec leurs analogues structuraux ICIS et TIP150, constituer une nouvelle superfamille de protéines associées aux microtubules. Localisées dans différents compartiments intracellulaires, les protéines ATIP pourraient contribuer aux fonctions essentielles de la cellule (mitose, trafic, signalisation) en régulant la dynamique des microtubules. L'étude moléculaire des membres de cette superfamille devrait permettre de révéler de nouveaux aspects du rôle des microtubules aux niveaux cérébral, cardiovasculaire et tumoral. $\diamond$ ATIP, a novel superfamily of microtubule-associated proteins 


\section{CONFLIT D'INTERÊTS}

Les auteurs déclarent n'avoir aucun conflit d'intérêt concernant les données publiées dans cet article.

\section{RÉFÉRENCES}

1. Arnal I, Sassoon I, Tournebize R. Dynamique du fuseau : vers une cible anti-cancéreuse. Med Sci (Paris) 2002 ; 18 : 1227-35.

2. Etienne-Manneville $S$. From signaling pathways to microtubule dynamics: the key players. Curr Opin Cell Biol 2010 ; 22 : 104-11.

3. Rodrigues-Ferreira S, Di Tommaso A, Dimitrov A, et al. 8p22 MTUS1 gene product ATIP3 is a novel anti-mitotic protein underexpressed in invasive breast carcinoma of poor prognosis. PLoS One 2009 ; 4 : e7239.
4. Rodrigues-Ferreira S, Nahmias C. An ATIPical family of angiotensin II AT2 receptor-interacting proteins. Trends Endocrinol Metab 2010 ; 21 : 684-90.

5. Di Benedetto M, Bièche I, Deshayes F, et al. Structural organization and expression of human MTUS1, a candidate $8 \mathrm{p} 22$ tumor suppressor gene encoding a family of angiotensin II AT2 receptor-interacting proteins, ATIP. Gene 2006 ; 380 : 127-36.

6. Ohi R, Coughlin ML, Lane WS, Mitchison TJ. An inner centromere protein that stimulates the microtubule depolymerizing activity of a Kinl kinesin. Dev Cell $2003 ; 5: 309-21$.

7. Knowlton AL, Vorozhko VV, Lan W, et al. ICIS and Aurora $B$ coregulate the microtubule depolymerase Kif2a. Curr Biol $2009 ; 19$ : 758-63.

8. Jiang K, Wang J, Liu J, et al. TIP150 interacts with and targets MCAK at the microtubule plus ends. EMBO Rep $2009 ; 10: 857-65$.

\section{NOUVELLE}

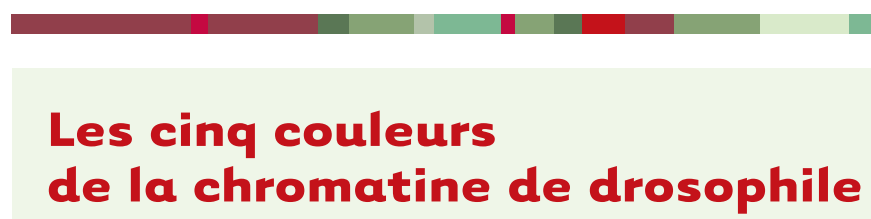

Guillaume Filion

\section{La chromatine et ses dérivés}

Depuis son apparition en 1882 [1], le terme « chromatine » a changé de définition à de nombreuses reprises. Ces définitions ont toutes eu pour fonction de mettre un nom sur un objet dont la nature, la structure, les propriétés et les fonctions n'ont jamais été parfaitement comprises. Par exemple, la dichotomie entre euchromatine et hétérochromatine, initialement établie par microscopie électronique, désignait l'hétérochromatine comme la fraction compacte du matériel nucléaire d'une cellule en interphase. La définition actuelle obéit aux exigences de la génomique: l'hétérochromatine est volontiers définie par sa nature répétitive ou l'absence d'expression des gènes qui y sont codés. Ainsi, chacune des deux définitions élude les mécanismes fondamentaux de la propriété sur laquelle elle est basée. Malgré la nature éminemment floue du concept de chromatine, la communauté scientifique ne fait preuve ni de dogmatisme, ni de naïveté quant à son usage. Le terme garde son aspect indéfini avant tout pour des raisons expérimentales. Il est clair depuis plus d'une quinzaine d'années que la dichotomie naïve entre euchromatine et hétérochromatine est le fruit de l'inefficacité des méthodes utilisées pour interroger les propriétés du matériel nucléaire in vivo. II n'est donc pas surprenant que les termes changent à nouveau de sens avec l'essor des analyses in vivo à l'échelle du génome. Grâce aux technologies récentes, nous avons cartographié les sites de liaison de 53 protéines de la chromatine sur le génome de la drosophile [2]. Pour cela nous avons utilisé un modèle uniforme de cellules en culture et une méthode de cartographie in vivo appelée DamID. Le principe de la méthode repose sur l'activité de l'enzyme Dam (DNA adenine methyltransferase). Dam dépose une empreinte sur I'ADN, absente des génomes eucaryotes, qui peut être détectée par des enzymes de restriction. En fusionnant une protéine à Dam, il est possible de restreindre la méthylation de I'ADN aux sites de liaison de cette protéine in vivo, ce qui permet ensuite de les iden-
9. Nouet S, Amzallag N, Li JM, et al. Trans-inactivation of receptor tyrosine kinases by novel angiotensin II AT2 receptor-interacting protein, ATIP. J Biol Chem 2004 ; $279: 28989-97$.

10. Wruck C, Funke-Kaiser H, Pufe T, et al. Regulation of transport of the angiotensin AT2 receptor by a novel membrane-associated golgi protein. Arterioscler Thromb Vasc Biol $2005 ; 25: 57-64$.

11. Seibold S, Rudroff C, Weber M, et al. Identification of a new tumor suppressor gene located at chromosome 8p21.3-22. FASEBJ 2003; $17: 1180-2$.
The Netherlands cancer institute, Gene regulation (B4),

Plesmanlaan 121,

1066CX Amsterdam,

Pays Bas.

g.filion@nki.nl

tifier (Figure 1). Donnant des résultats comparables à ceux de la précipitation de la chromatine (ChIP), la technologie DamID jouit d'un avantage: elle peut être appliquée de façon systématique sans recours au développement d'anticorps spécifiques, ce qui permet une augmentation conséquente du débit. Les cartes de liaison à haute résolution de ces 53 protéines nous ont permis de donner le premier aperçu de la chromatine à cette échelle dans une cellule eucaryote.

\section{Hétérogénéité et simplicité}

\section{de la chromatine de drosophile}

La première surprise de cette étude est I'hétérogénéité de la chromatine de drosophile. On pourrait s'attendre à une répartition plus ou moins uniforme des évènements de liaison sur la molécule d'ADN. Or, la majeure partie du génome est liée par un petit nombre de protéines très abondantes, telles que l'histone $\mathrm{Hl}$, alors qu'une petite fraction $\mathrm{du}$ génome est liée par un très grand nombre de protéines différentes. La seconde surprise est la faible complexité de la 